\title{
The Co-Expression of Melanoma-Antigen Family a Proteins and New York Esophageal Squamous Cell Carcinoma-I in Breast Cancer: A Pilot Study
}

\author{
Yu-Xin Wang ${ }^{l, *}$ \\ Feng-Lian $\mathrm{Li}^{1}{ }^{1, *}$ \\ $\mathrm{Li}$-Xin $\mathrm{Du}{ }^{\mathrm{l}}$ \\ Jun-Fang Liu $^{2}$ \\ Li-Gang Huo' \\ Shu-Qing $\mathrm{Li}^{3}$ \\ Bin Tian ${ }^{1}$
}

\begin{abstract}
'Department of Clinical Laboratory, Hengshui Fifth People's Hospital,

Hengshui City, 053000, People's Republic of China; ${ }^{2}$ Department of Pathology, Hengshui Fifth People's Hospital, Hengshui City, 053000, People's Republic of China; ${ }^{3}$ Department of cardiothoracic surgery, Hengshui Fifth People's Hospital, Hengshui City, 053000, People's Republic of China
\end{abstract}

*These authors contributed equally to this work
Correspondence: Feng-Lian Li

Department of Clinical Laboratory, Hengshui Fifth People's Hospital, No. 1638

Shengli West Road, Hengshui City, Hebei

Province, 053000, People's Republic of

China

Tel +86 3I8-23I6645

Email fenglianlidr@163.com
Objective: The aim of this study was to quantify the expression of melanoma-antigen family A proteins (MAGE-A) and New York esophageal squamous cell carcinoma-1 (NYESO-1) in breast cancer and establish the prognosis of breast cancer patients with MAGE-A and NY-ESO-1 co-expression.

Methods: A total of 122 patients with breast cancer were recruited for this study. Their clinicopathological data were collected retrospectively, and the MAGE-A and NY-ESO-1 expressions in paraffin-embedded specimens from the 122 patients were evaluated using immunohistochemical analysis. In addition, the survival states of the patients were recorded. Results: Fifty-four patients (44.26\%) were MAGE-A positive and 46 (37.70\%) were NYESO-1 positive. Interestingly, 22 of the 46 NY-ESO-1-positive cases co-expressed MAGE-A. The expression of MAGE-A was positively associated with estrogen-receptor status $\left(\chi^{2}=\right.$ $4.026, \mathrm{p}=0.045)$ and human epidermal growth factor receptor 2 status $\left(\chi^{2}=5.482, \mathrm{p}=\right.$ $0.019)$, while the expression of NY-ESO-1 was positively associated with p53 expression $\left(\chi^{2}\right.$ $=4.541, \mathrm{p}=0.033)$. Of the 122 patients, the lowest survival rate was observed in patients with NY-ESO-1 (+)/MAGE-A (+), with a 5-year survival rate of $59.09 \%$ and a median survival of 97 months.

Conclusion: The results showed that MAGE-A and NY-ESO-1 were frequently expressed in breast cancer patients. The co-expression of MAGE-A and NY-ESO-1 occurred in about $18 \%$ of these patients, and it may indicate a poor prognosis.

Keywords: breast cancer, cancer/testis antigens, MAGE-A, NY-ESO-1, co-expression

\section{Introduction}

Breast cancer is the leading cause of cancer-related deaths in women. It has been reported that approximately 1,7000,000 new cases occur globally each year, ${ }^{1}$ and the incidence and mortality rates of breast cancer are expected to increase significantly in the next 5-10 years. ${ }^{2}$ Immunotherapy against tumor-associated antigens, using the body's immune cells to recognize tumor-related antigens, has become one of the most desirable treatment options for breast cancer patients. Of all the tumorassociated antigens, cancer/testis antigens (CTAs) are of particular interest as a potential target for immunotherapy because of their strong in vivo immunogenicity and unique expression patterns. ${ }^{3}$

CTAs belong to a group of proteins that are expressed in the developing embryo, and they are aberrantly re-expressed in malignancy, particularly in high-grade and advanced-stage tumors. ${ }^{4}$ Two of the most well-studied CTAs are the New York 
esophageal squamous cell carcinoma-1 (NY-ESO-1) and the melanoma-antigen family A proteins (MAGE-A), which are encoded respectively by the CTAGB1 and MAGEA family of genes located on the X chromosome. ${ }^{5}$ It has been reported that over-expressed NY-ESO-1 and/or MAGE-A could elicit potent T-cell responses. ${ }^{6,7}$ They have been shown to evoke spontaneous cytotoxic T-cell responses in melanoma, esophageal carcinoma, bladder cancer, and non-small-cell lung carcinoma. ${ }^{8,9}$ Therefore, NY-ESO-1 and MAGE-A are promising tumor-specific immunotherapeutic targets. 6,7

Several studies have evaluated MAGE-A and NYESO-1 expression in breast cancer. For example, Raghavendra et al found that MAGE-A and NY-ESO-1 were frequently expressed in triple-negative breast cancer ( $\sim 7 \%$ and $17 \%$ of cases, respectively). ${ }^{4}$ Matković et al conducted a retrospective study that included samples from 49 medullary breast cancer patients. They found that MAGE-A and NY-ESO-1 antigens were highly expressed in medullary breast cancer, and the expression of these CTAs may represent a marker of potential prognostic relevance in this type of cancer. ${ }^{10}$ Furthermore, a possible predictive role for CTAs was detected by Roguljic et al They found that CTAs from the MAGE family (MAGE-A1, multi-MAGE-A, and MAGE-A10) and NY-ESO-1 were associated with the histopathological predictive variables of breast ductal carcinoma in situ. ${ }^{11}$

The co-expression of MAGE-A and NY-ESO-1 in breast cancer has also been found in previous studies. ${ }^{4}$ However, the prognosis of patients with MAGE-A and NY-ESO-1 co-expression has not been explored previously. This study aimed to quantify the expression of MAGE-A and NY-ESO-1 CTAs in breast cancer and establish the prognosis of breast cancer patients with MAGE-A and NY-ESO-1 co-expression.

\section{Materials and Methods}

\section{Patients and Clinical Parameters}

A total of 122 patients with breast cancer were recruited from our hospital between March 2009 and March 2010. None of the patients underwent chemotherapy, radiotherapy, or endocrinotherapy prior to surgery. Written informed consent was provided by all the participants before enrollment, and the study protocol was approved by the Medical Ethics Committee of our hospital.
The clinicopathological data of the patients were retrospectively collected, including age, tumor size, clinical stage, histological grade, estrogen receptor (ER) status, progesterone receptor (PR) status, human epidermal growth factor receptor 2 (HER2) status, vascular endothelial growth factor (VEGF) status, tumor protein p53 status, and lymph-node metastasis status.

\section{Immunohistochemical Staining}

Deparaffinization of the paraffin-embedded tissue sections $(5 \mu \mathrm{m})$ was accomplished using xylene and graded alcohols. Antigen retrieval was conducted for 30 minutes using Bond epitope retrieval solution 2 (ethylenediaminetetraacetic acid, $\mathrm{pH}$ 9.0) followed by the incubation of the primary antibody for 30 minutes. The primary antibodies used in this study were NY-ESO-1 (1:100 dilution; Invitrogen Technologies, Grand Island, NY, USA) and MAGE-A (1:100 dilution; Santa-Cruz Biotech, Dallas, TX, USA). Immunohistochemical (IHC) staining for NYESO-1 and MAGE-A was performed on 4-micron thick formalin-fixed paraffin-embedded serial sections using an automated staining system (Leica Bond). Appropriate positive controls were included in each staining run (MAGE-A, placenta; NY-ESO-1, normal testicle). ${ }^{12}$

The NY-ESO-1 and MAGE staining intensity was assessed by two pathologists. Positivity for NY-ESO-1 and MAGE-A was defined by the membranous and/or cytoplasmic expression. Immunoreactivity for the MAGEA4 and NY-ESO-1 was scored in the following way: 0, no positive tumor cells (negative); $1+,<20 \%$ positive tumor cells (mild reaction); $2+, 21-50 \%$ positive tumor cells (moderate reaction); and $3+,>50 \%$ positive tumor cells (strong reaction). The immunoreactivity scores were presented as either negative or positive, with positive including mild, moderate, and strong reactions. ${ }^{12,13}$

\section{Follow-Up}

Based on their NY-ESO-1 and MAGE-A status, the 122 breast cancer patients were divided into the following four groups: NY-ESO-1 (-)/MAGE-A $\quad(-), \quad$ NY-ESO-1 $(-)$ /MAGE-A (+), NY-ESO-1 (+)/MAGE-A (-), and NYESO-1 $(+)$ /MAGE-A $(+)$. The patients in the four groups were followed up via telephone, outpatient services, or hospitalization to record their survival state up to March 2021. Overall survival (OS) was defined as the time from the day of diagnosis to death for any reason or the final follow-up, if death had not occurred before then. 


\section{Statistical Analysis}

SPSS v20.0 software (IBM Corp.) was used to analyze the data. The associations between MAGE-A, NY-ESO-1, and the clinicopathological parameters were evaluated through the chi-square test and Fisher's exact test. The KaplanMeier method was used to estimate the OS time of the patients, with Log rank tests being performed to assess significance. $\mathrm{P}<0.05$ was considered to indicate a statistically significant difference.

\section{Results}

\section{Patient Characteristics}

The median age of the 122 patients at diagnosis was 58 years old (range: 34-76), and the median follow-up period was 101 months. Most of the patients were at clinical stage grade 2 $(62.30 \%)$ and at histological stage grade $2(60.66 \%)$, while lymph-node metastasis had occurred in $49.18 \%$ of the patients. In addition, $63.93 \%$ of the patients were ER positive, $62.30 \%$ were PR positive, $42.62 \%$ were HER 2 positive, $90.16 \%$ were VEGF positive, and $40.98 \%$ were $\mathrm{p} 53$ positive. The characteristics of the 122 patients are shown in detail in Table 1.

\section{NY-ESO-I and MAGE-A Expression}

MAGE-A and NY-ESO-1 showed homogeneous staining, with positivity in both cytoplasmic and nuclear tumor cell compartments (see Figure 1). The results showed that 54 cases $(44.26 \%)$ were MAGE-A positive and 46 patients (37.70\%) were NY-ESO-1 positive. Interestingly, 22 of the 46 NY-ESO-1-positive cases co-expressed MAGE-A. Thus, the 122 breast cancer patients were divided into four groups: the NY-ESO-1 (-)/MAGE-A (-) group (44 cases), the NY-ESO-1 (-)/MAGE-A (+) group (32 cases), the NY-ESO-1 (+)/MAGE-A (-) group (24 cases), and the NY-ESO-1 (+)/MAGE-A (+) group (22 cases).

\section{Associations Between MAGE-A, NY-ESO- $\mathrm{I}$, and the Clinicopathological Parameters} As shown in Figure 2A, the expression of MAGE-A was positively associated with ER status $\left(\chi^{2}=4.026, p=\right.$ $0.045)$ and HER2 status $\left(\chi^{2}=5.482, p=0.019\right)$. However, the associations between MAGE-A expression, PR expression, p53 status, VEGF expression, and lymphnode metastasis status were not statistically significant. As shown in Figure 2B, the expression of NY-ESO-1 was not associated with any of the clinicopathological parameters shown above (ER, HER2, PR, VEGF, or lymph-node metastasis $)$ apart from $\mathrm{p} 53\left(\chi^{2}=4.541, \mathrm{p}=0.033\right)$.
Table I Clinical Data of the I22 Breast Cancer Patients

\begin{tabular}{|c|c|c|}
\hline Clinical Parameters & Cases (n) & Percent (\%) \\
\hline \multicolumn{3}{|l|}{ Age (years) } \\
\hline$<55$ & 66 & 54.10 \\
\hline$\geq 55$ & 56 & 45.90 \\
\hline \multicolumn{3}{|l|}{ Clinical stage } \\
\hline I & 20 & 16.39 \\
\hline II & 76 & 62.30 \\
\hline III & 26 & 21.31 \\
\hline \multicolumn{3}{|l|}{ Histological grade } \\
\hline I & 16 & 13.11 \\
\hline II & 74 & 60.66 \\
\hline III & 32 & 26.23 \\
\hline \multicolumn{3}{|l|}{ Size of tumor $(\mathrm{cm})$} \\
\hline$\leq 2$ & 14 & 11.48 \\
\hline$>2$ or $\leq 5$ & 24 & 19.67 \\
\hline$>5$ & 84 & 68.85 \\
\hline \multicolumn{3}{|l|}{$\begin{array}{l}\text { Metastatic state of } \\
\text { lymph node }\end{array}$} \\
\hline No & 62 & 50.82 \\
\hline Yes & 60 & 49.18 \\
\hline \multicolumn{3}{|l|}{$\begin{array}{l}\text { Estrogen receptor } \\
\text { status }\end{array}$} \\
\hline- & 44 & 36.07 \\
\hline+ & 78 & 63.93 \\
\hline \multicolumn{3}{|l|}{$\begin{array}{l}\text { Progesterone } \\
\text { receptor status }\end{array}$} \\
\hline- & 46 & 37.70 \\
\hline+ & 76 & 62.30 \\
\hline \multicolumn{3}{|l|}{ P53 status } \\
\hline- & 72 & 59.02 \\
\hline+ & 50 & 40.98 \\
\hline \multicolumn{3}{|l|}{$\begin{array}{l}\text { Human epidermal } \\
\text { growth factor } \\
\text { receptor } 2 \text { status }\end{array}$} \\
\hline- & 70 & 57.38 \\
\hline+ & 52 & 42.62 \\
\hline \multicolumn{3}{|l|}{$\begin{array}{l}\text { Vascular endothelial } \\
\text { growth factor status }\end{array}$} \\
\hline- & 12 & 9.84 \\
\hline+ & 110 & 90.16 \\
\hline
\end{tabular}

Of the 22 cases with NY-ESO-1 and MAGE-A coexpression, 15 (68.18\%) were ER positive, 14 (63.64\%) were PR positive, 17 (77.27\%) were HER2 positive, 16 (70.73\%) were 553 positive, and $9(40.91 \%)$ had lymph-node metastasis. The cases with NY-ESO-1 and MAGE-A co-expression had high expression rates of HER2, ER, PR, and p53. 




Figure I Positive expression of MAGE-A/NY-ESO-I in breast cancer tissues.

\section{Comparison of Survival Times}

In this study, the median follow-up period was 101 months. The survival curves of the four groups are shown in Figure 3. The lowest survival rate was observed in the NY-ESO-1 $(+)$ /MAGE-A (+) group with a 5-year survival rate of $59.09 \%$ and a median survival of 97 months. In contrast, the highest survival rate was seen in the NY-ESO-1 (-)/MAGE-A (-) group, with a 5-year survival rate of $86.19 \%$ and a median survival of 119 months. In addition, the median survival for the NY-ESO -1 (-)/MAGE-A (+) and NY-ESO-1 (+)/MAGE-A (-) groups were 112 months and 103 months, respectively. However, the survival time among the four groups was not significantly different $(\mathrm{p}=0.184)$.

\section{Discussion}

This study investigated the expression of MAGE-A and NYESO-1 in breast cancer and found that MAGE-A and NY-ESO-1 were expressed frequently. Furthermore, MAGE-A and NY-ESO-1 co-expression existed in about

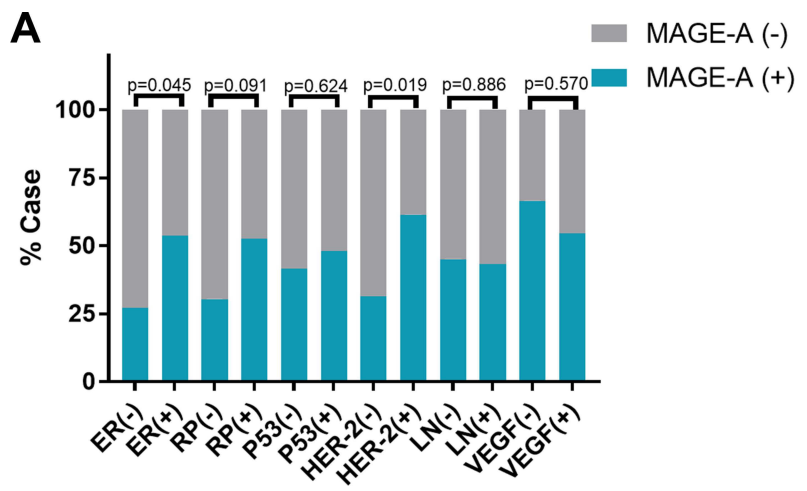

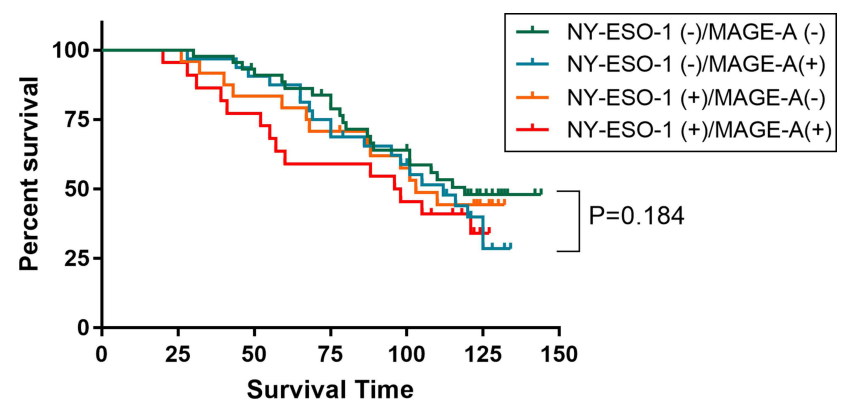

Figure 3 Kaplan-Meier survival analysis of the whole follow-up cohort.

$18 \%$ of the patients and tended to indicate a poor prognosis.

In previous studies, the expression of MAGE-A and NYESO-1 in breast cancer ranged from $17 \%$ to $74 \%$ and from $2 \%$ to $40 \%$, respectively. ${ }^{4,13,14}$ The results of this study showed that, in line with previous studies, $44.26 \%$ of patients were MAGE-A positive and $37.70 \%$ of patients were NYESO-1 positive. ${ }^{4,13,14}$ Kerkar et al conducted a systematic IHC analysis of 3668 cancer cases and found that the expression of MAGE-A was significantly higher than that of NYESO-1 in the majority of cancers. ${ }^{5}$ The present study also found that there were more MAGE-A-positive breast cancer patients than NY-ESO-1-positive. An extensive IHC analysis of the expression of eight CTAs in 454 invasive ductal carcinomas revealed that the co-expression of CTAs (including MAGE-A and NY-ESO-1) was frequent in breast cancer, especially in ER-negative tumors. ${ }^{15}$ The findings of the present study also demonstrate that MAGE-A and NY-ESO-1 co-expression is frequent in breast cancer; 22 of the 122 (18.03\%) patients co-expressed MAGE-A and NY-ESO-1. However, in this study, patients with MAGE-A and NY-ESO -1 co-expression had high ER-positive, PR-positive, and

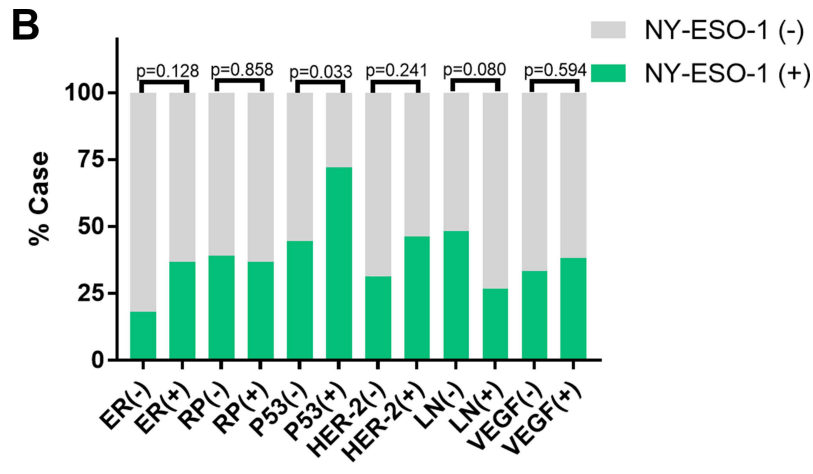

Figure 2 (A and B) Chi-square or Fisher's exact test analysis of associations between expression of MAGE-A/NY-ESO-I and clinical diagnostic or experimental biomarkers. Abbreviations: ER, estrogen receptor; PR, progesterone receptor; HER-2, human epidermal growth factor receptor 2; LN, lymph node; MAGE-A, melanoma-antigen family A protein; NY-ESO-I, New York esophageal squamous cell carcinoma-I. 
HER2 rates, which is not consistent with previous studies. ${ }^{4,15}$ The differences between this study and other studies are difficult to explain, but the following factors may have affected the results: 1) the criteria for IHC positivity, 2) the sample size, and 3) the profiles of the breast cancer patients.

The expression of MAGE-A family and NY-ESO-1 CTAs is often regarded as an independent marker for poor survival in solid tumors. ${ }^{16,17}$ Both MAGE-A and NYESO-1 have been demonstrated to be associated with histopathological predictive variables. In a study conducted by Roguljic et al, MAGE-A was shown to be significantly associated with a higher expression of ER and a higher tumor grade and NY-ESO-1 with a larger tumor size, the expression of tumor-infiltrating lymphocytes, and R1 resection. ${ }^{11}$ In a study conducted by Raghavendra et al, the expression of both MAGE-A and NY-ESO-1 CTAs was positively associated with c-Kit, p53, and the mitosis independent expression of threonine and tyrosine kinase. ${ }^{4}$ The present study found that the expression of MAGE-A was positively associated with ER and HER2 status, while NY-ESO-1 was positively associated with p53 status. The HER2 gene is amplified in $15-20 \%$ of invasive breast cancers, and its amplification is closely linked to HER2 protein overexpression. ${ }^{18}$ HER2 amplification (positive) is a poor prognostic factor associated with a high rate of recurrence and mortality for breast cancer. ${ }^{18}$ Due to the association with HER2 coexpression, the findings of this study suggest that MAGE-A-positive tumors also have a poor prognosis. As it controls the cell cycle, DNA replication, and uncontrolled cell division that take place while a tumor is growing, p53 is known as a tumor suppressor protein. When it mutates or aggregates, it loses its function, resulting in tumor progression and growth. ${ }^{19}$ Mutations in p53 can result in a stable non-functional protein that accumulates in the nucleus, giving rise to an IHC phenotype mimicking overexpression. ${ }^{20}$ In this study, the positive association between NY-ESO-1 and p53 expression indicates that NY-ESO-1-positive tumors have a proliferative advantage.

Although MAGE-A and NY-ESO-1 belong to the same group of CTAs, they may affect the prognosis of breast cancer through different mechanisms. As a result, patients with a co-expression of MAGE-A and NY-ESO-1 seem to be more difficult to treat. Veit et al found MAGE-A and NY-ESO-1 co-expression was associated with the reduced OS of patients with adenoid cystic carcinoma. ${ }^{21}$ As expected, in this study, patients with MAGE-A and NY-
ESO-1 co-expression had the lowest 5-year survival rate and shortest median survival time. This raises the possibility that therapies simultaneously targeting multiple CTAs may elicit more efficient antitumor responses than singleantigen approaches.

This study had several limitations. First, MAGE-A constitutes a protein family, and 12 subtypes of MAGEA have been reported. Each MAGE-A protein contains the MAGE-A homology domain, but there are some differences in the structures and functions of these proteins. ${ }^{22}$ However, this study did not consider the MAGE-A subtypes in breast cancer. Second, the sample size of this study was small, so further studies with larger cohorts are needed. Third, $42.62 \%$ of the patients in this study were HER2 positive. This is a higher proportion of HER2positive patients than reported in previous studies, ${ }^{23}$ which may be the result of an insufficient number of patients. Finally, the impact of CTA expression on the OS of breast cancer patients could not be evaluated, as the number of patients who died during follow-up was small.

In conclusion, this study showed that MAGE-A and NY-ESO-1 were frequently expressed in breast cancer patients, and the co-expression of MAGE-A and NYESO-1 occurred in about $18 \%$ of these patients. It would appear that MAGE-A and NY-ESO-1 co-expression indicates a poor prognosis for breast cancer patients.

\section{Data Sharing Statement}

All data generated or analyzed during this study are included in this published article.

\section{Statement of Ethics}

This study was conducted in accordance with the Declaration of Helsinki and approved by the ethics committee of Hengshui Fifth People's Hospital. Written informed consent was obtained from all participants.

\section{Author Contributions}

All authors made a significant contribution to the work reported, whether that is in the conception, study design, execution, acquisition of data, analysis and interpretation, or in all these areas; took part in drafting, revising or critically reviewing the article; gave final approval of the version to be published; have agreed on the journal to which the article has been submitted; and agree to be accountable for all aspects of the work. 


\section{Funding}

Expression of tumor antigen MAGE-A family in breast cancer and its role in immunotherapy; No. $2018014006 Z$.

\section{Disclosure}

The authors declare that they have no conflicts of interest for this work.

\section{References}

1. Anastasiadi Z, Lianos GD, Ignatiadou E, Harissis HV, Mitsis M. Breast cancer in young women: an overview. Updates Surg. 2017;69(3):313-317. doi:10.1007/s13304-017-0424-1

2. Greaney ML, Sprunck-Harrild K, Ruddy KJ, et al. Study protocol for young \& strong: a cluster randomized design to increase attention to unique issues faced by young women with newly diagnosed breast cancer. BMC Public Health. 2015;15(1):37. doi:10.1186/s12889-015-1346-9

3. Zhang Y, Zhang Y, Zhang L. Expression of cancer-testis antigens in esophageal cancer and their progress in immunotherapy. $J$ Cancer Res Clin Oncol. 2019;145(2):281-291. doi:10.1007/s00432-01902840-3

4. Raghavendra A, Kalita-de Croft P, Vargas AC, et al. Expression of MAGE-A and NY-ESO-1 cancer/testis antigens is enriched in triple-negative invasive breast cancers. Histopathology. 2018;73 (1):68-80. doi:10.1111/his.13498

5. Kerkar SP, Wang Z-F, Lasota J, et al. MAGE-A is more highly expressed than NY-ESO-1 in a systematic immunohistochemical analysis of 3668 cases. $J$ Immunother. 2016;39(4):181-187. doi:10.1097/CJI.0000000000000119

6. Schooten E, Di Maggio A, van Bergen En Henegouwen PMP, Kijanka MM. MAGE-A antigens as targets for cancer immunotherapy. Cancer Treat Rev. 2018;67:54-62. doi:10.1016/j. ctrv.2018.04.009

7. Raza A, Merhi M, Inchakalody VP, et al. Unleashing the immune response to NY-ESO-1 cancer testis antigen as a potential target for cancer immunotherapy. J Transl Med. 2020;18(1):140. doi:10.1186/ s12967-020-02306-y

8. Jäger E, Stockert E, Zidianakis Z, et al. Humoral immune responses of cancer patients against "Cancer-Testis" antigen NY-ESO-1: correlation with clinical events. Int J Cancer. 1999;84(5):506-510. doi:10.1002/ (sici)1097-0215(19991022)84:5<506::aid-ijc10>3.0.co;2-6

9. van der Bruggen $\mathrm{P}$, Traversari C, Chomez P, et al. A gene encoding an antigen recognized by cytolytic $\mathrm{T}$ lymphocytes on a human melanoma. Science. 1991;254(5038):1643-1647. doi:10.1126/science.1840703

10. Matković B, Juretić A, Spagnoli GC, et al. Expression of MAGE-A and NY-ESO-1 cancer/testis antigens in medullary breast cancer: retrospective immunohistochemical study. Croat Med J. 2011;52(2):171-177. doi:10.3325/cmj.2011.52.171
11. Roguljic A, Spagnoli G, Juretic A, Sarcevic B, Banovic M, Beketic Oreskovic L. Possible predictive role of cancer/testis antigens in breast ductal carcinoma in situ. Oncol Lett. 2018;16(6):7245-7255. doi: 10.3892/ol.2018.9544

12. Pourmaleki M, Young JH, Socci ND, et al. Extramammary Paget disease shows differential expression of B7 family members B7-H3, B7-H4, PD-L1, PD-L2 and cancer/testis antigens NY-ESO-1 and MAGE-A. Oncotarget. 2019;10(58):6152-6167. doi:10.18632/ oncotarget. 27247

13. Bandić D, Juretić A, Sarcević B, et al. Expression and possible prognostic role of MAGE-A4, NY-ESO-1, and HER-2 antigens in women with relapsing invasive ductal breast cancer: retrospective immunohistochemical study. Croat Med J. 2006;47(1):32-41.

14. Badovinac ČT, Spagnoli G, Juretić A, Jakić-Razumović J, Podolski P, Šarić N. High expression of MAGE-A10 cancer-testis antigen in triple-negative breast cancer. Med Oncol. 2012;29(3):1586-1591. doi:10.1007/s12032-011-0120-9

15. Chen YT, Ross DS, Chiu R, et al. Multiple cancer/testis antigens are preferentially expressed in hormone-receptor negative and high-grade breast cancers. PLoS One. 2011;6(3):e17876. doi:10.1371/journal. pone. 0017876

16. Laban S, Atanackovic D, Luetkens T, et al. Simultaneous cytoplasmic and nuclear protein expression of melanoma antigen-A family and NY-ESO-1 cancer-testis antigens represents an independent marker for poor survival in head and neck cancer. Int $J$ Cancer. 2014;135 (5):1142-1152. doi:10.1002/ijc.28752

17. Tio D, Willemsen M, Krebbers G, et al. Differential expression of cancer testis antigens on lentigo maligna and lentigo maligna melanoma. Am J Dermatopathol. 2020;42(8):625-627. doi:10.1097/ DAD.0000000000001607

18. Ahn S, Woo JW, Lee K, Park SY. HER2 status in breast cancer: changes in guidelines and complicating factors for interpretation. J Pathol Transl Med. 2020;54(1):34-44. doi:10.4132/jptm.2019. 11.03

19. Kanapathipillai M. Treating p53 mutant aggregation-associated cancer. Cancers. 2018;10(6):154. doi:10.3390/cancers10060154

20. Vousden KH, Lane DP. p53 in health and disease. Nat Rev Mol Cell Biol. 2007;8(4):275-283. doi:10.1038/nrm2147

21. Veit JA, Heine D, Thierauf J, et al. Expression and clinical significance of MAGE and NY-ESO-1 cancer-testis antigens in adenoid cystic carcinoma of the head and neck. Head Neck. 2016;38 (7):1008-1016. doi:10.1002/hed.24403

22. Caballero OL, Shousha S, Zhao Q, Simpson AJG, Coombes RC, Neville AM. Expression of cancer/testis genes in ductal carcinoma in situ and benign lesions of the breast. Oncoscience. 2013;1 (1):14-20. doi:10.18632/oncoscience.4

23. Sendur MA, Aksoy S, Altundag K. Pertuzumab in HER2-positive breast cancer. Curr Med Res Opin. 2012;28(10):1709-1716. doi:10.1185/03007995.2012.728132

\section{Publish your work in this journal}

Cancer Management and Research is an international, peer-reviewed open access journal focusing on cancer research and the optimal use of preventative and integrated treatment interventions to achieve improved outcomes, enhanced survival and quality of life for the cancer patient.
The manuscript management system is completely online and includes a very quick and fair peer-review system, which is all easy to use. Visit http://www.dovepress.com/testimonials.php to read real quotes from published authors. 